\title{
Effect of small-vessel disease on cognitive trajectory after atrial fibrillation-related ischaemic stroke or TIA
}

\author{
Gargi Banerjee ${ }^{1}$ (1) $\cdot$ Edgar Chan $^{2} \cdot$ Gareth Ambler $^{3} \cdot$ Duncan Wilson $^{1,4} \cdot$ Lisa Cipolotti $^{2} \cdot$ Clare Shakeshaft $^{1}$. \\ Hannah Cohen ${ }^{5} \cdot$ Tarek Yousry $^{6}$. Gregory Y. H. Lip ${ }^{7,8} \cdot$ Keith W. Muir $^{9} \cdot$ Martin M. Brown ${ }^{1} \cdot$ Hans Rolf Jäger $^{6}$. \\ David J. Werring ${ }^{1} \cdot$ The CROMIS-2 collaborators
}

Received: 7 January 2019 / Revised: 16 February 2019 / Accepted: 19 February 2019 / Published online: 7 March 2019

(c) The Author(s) 2019

\begin{abstract}
Post-stroke dementia is common but has heterogenous mechanisms that are not fully understood, particularly in patients with atrial fibrillation (AF)-related ischaemic stroke or TIA. We investigated the relationship between MRI small-vessel disease markers (including a composite cerebral amyloid angiopathy, CAA, score) and cognitive trajectory over 12 months. We included patients from the CROMIS-2 AF study without pre-existing cognitive impairment and with Montreal Cognitive Assessment (MoCA) data. Cognitive impairment was defined as MoCA $<26$. We defined "reverters" as patients with an "acute" MoCA (immediately after the index event) score $<26$, who then improved by $\geq 2$ points at 12 months. In our cohort $(n=114), 12$-month MoCA improved overall relative to acute performance (mean difference 1.69 points, 95\% CI 1.03-2.36, $p<0.00001) .12$-month cognitive impairment was associated with increasing CAA score (per-point increase, adjusted OR $4.09,95 \%$ CI 1.36-12.33, $p=0.012)$. Of those with abnormal acute MoCA score $(n=66), 59.1 \%(n=39)$ were "reverters". Non-reversion was associated with centrum semi-ovale perivascular spaces (per-grade increase, unadjusted OR 1.83, 95\% CI 1.06-3.15, $p=0.03$ ), cerebral microbleeds (unadjusted OR 10.86, 95\% CI 1.22-96.34, $p=0.03$ ), and (negatively) with multiple ischaemic lesions at baseline (unadjusted OR $0.11,95 \%$ CI $0.02-0.90, p=0.04$ ), as well as composite small-vessel disease (per-point increase, unadjusted OR 2.91, 95\% CI 1.23-6.88, $p=0.015$ ) and CAA (per-point increase, unadjusted OR 6.71, 95\% CI 2.10-21.50, $p=0.001$ ) scores. In AF-related acute ischaemic stroke or TIA, cerebral small-vessel disease is associated both with cognitive performance at 12 months and failure to improve over this period.
\end{abstract}

Keywords Atrial fibrillation · Brain ischaemia $\cdot$ Cerebral small-vessel disease $\cdot$ Cognitive impairment $\cdot$ Ischaemic stroke . Transient ischaemic attack (TIA)

David J. Werring

d.werring@ucl.ac.uk

1 Department of Brain Repair and Rehabilitation, Stroke Research Centre, UCL Queen Square Institute of Neurology and National Hospital for Neurology and Neurosurgery, Russell Square House, 10-12 Russell Square, London WC1B 5EH, UK

2 Department of Neuropsychology, National Hospital for Neurology and Neurosurgery, Queen Square, London, UK

3 Department of Statistical Science, University College London, Gower Street, London, UK

4 New Zealand Brain Research Institute, Christchurch, New Zealand

5 Haemostasis Research Unit, Department of Haematology, University College London, 51 Chenies Mews, London, UK
6 Lysholm Department of Neuroradiology and the Neuroradiological Academic Unit, Department of Brain Repair and Rehabilitation, UCL Queen Square Institute of Neurology, Queen Square, London, UK

7 Liverpool Centre for Cardiovascular Science, University of Liverpool and Liverpool Heart and Chest Hospital, Liverpool, UK

8 Aalborg Thrombosis Research Unit, Department of Clinical Medicine, Aalborg University, Aalborg, Denmark

9 Institute of Neuroscience and Psychology, University of Glasgow, Queen Elizabeth University Hospital, Glasgow, UK 


\section{Introduction}

Post-stroke dementia is common [30] but has heterogenous mechanisms that are not fully understood. Early post-stroke dementia (within 6 months) is associated with factors relating to brain resilience and the index stroke lesion, whereas delayed-onset post-stroke dementia is more associated with cerebral small-vessel diseases [23]. This appears to be the case for both ischaemic and haemorrhagic stroke; dementia after ischaemic intracerebral events (stroke or transient ischaemic attacks, TIA) is associated with white-matter hyperintensities, lacunes and cortical atrophy $[1,24]$, and markers of cerebral amyloid angiopathy (CAA) are associated with dementia after intracerebral haemorrhage [25]. The contribution of small-vessel disease to post-stroke dementia in patients with atrial fibrillation (AF)-related ischaemic stroke or TIA is unknown. AF is recognised as an independent risk factor for dementia [20]; proposed mechanisms include silent brain infarction from recurrent embolization, cerebral hypoperfusion, chronic inflammation and endothelial dysfunction, or progression of pre-existing cerebrovascular or neurodegenerative processes [31], but information on the imaging associations of post-stroke dementia in this context remains limited.

The natural history of post-stroke dementia is further complicated by the fact that cognitive performance immediately after a stroke might not be representative of later cognition, as performance might improve; this occurs both acutely, where the initial assessment might be influenced by delirium, but also over longer time periods [2, 10, 17, 19, 29]. The Montreal Cognitive Assessment (MoCA) appears to be a sensitive cognitive screen for identifying these changes [34], and there has been recent interest in identifying the characteristics of so-called "reverters", who demonstrate improvements in their cognitive performance over time [27, 34, 38, 43].

We investigated cognitive trajectory in patients with AF-related ischaemic stroke or TIA. Our objectives were: (1) to describe the changes in MoCA that occur between acute (immediately post ischaemic event) and 12 month assessment, (2) to investigate the clinical and radiological associations of MoCA-defined cognitive impairment at 12 months, and (3) to describe the clinical and imaging profile of reverters and non-reverters.

\section{Materials and methods}

\section{Patient selection}

This is a subgroup analysis of the CROMIS-2 AF study, the protocol for which has been described [7, 41]. Briefly, this was a multi-centre prospective observational study of adults (aged 18 years or above) presenting with ischaemic cardioembolic stroke or TIA with non-valvular atrial fibrillation (confirmed by electrocardiography), who were eligible to start anticoagulation following their ischaemic event [7, 41]. Patients who could not have an MRI scan, had contraindications to anticoagulation, or had previously received therapeutic anticoagulation, were excluded [7, 41]. The study was approved by the National Research Ethics Service (IRAS reference 10/H0716/61), and written informed consent was obtained for each patient.

We excluded patients with known documented diagnosis of dementia or cognitive impairment, or 16-item IQCODE (Informant Questionnaire for Cognitive Decline in the Elderly) score $>3.3$ [16] at study entry (Fig. 1). We compared characteristics of eligible (those with 12 month MoCA data) and excluded patients (those without) to assess selection bias.

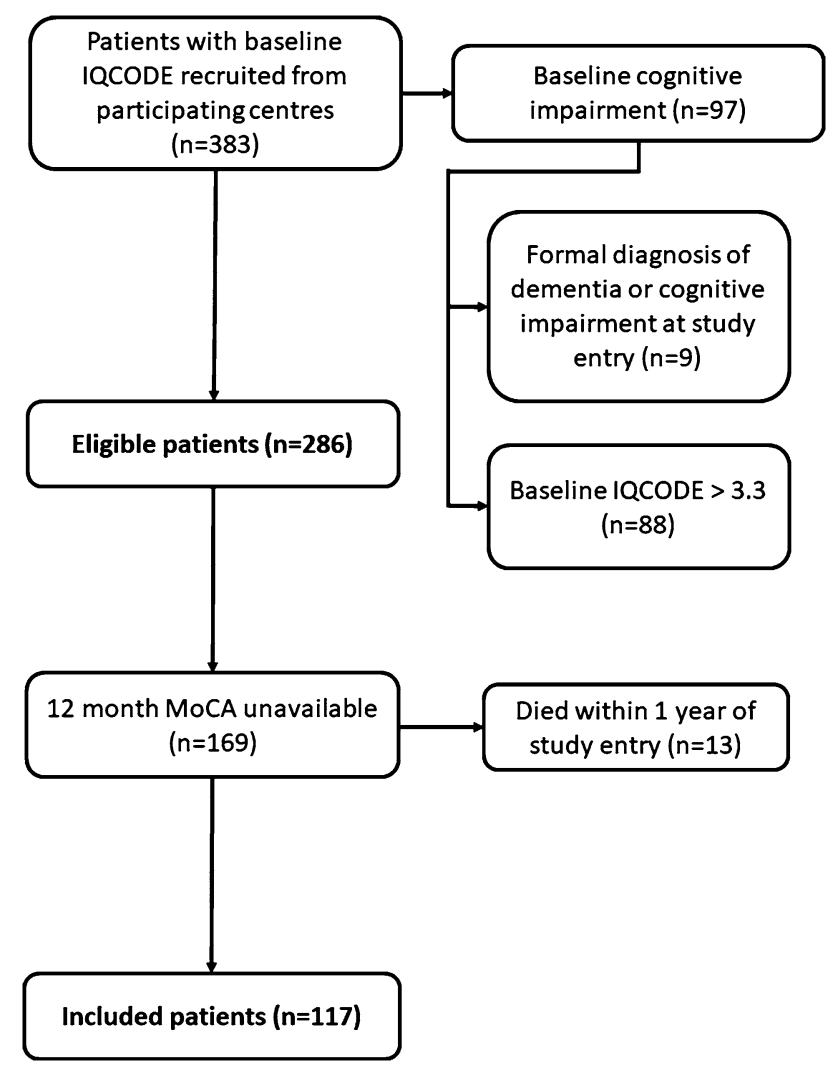

Fig. 1 Description of study population 


\section{Cognitive measures}

The "acute" MoCA was collected immediately after the index ischaemic event. All participating centres were invited to collect additional MoCA data at 12 months ("12 month MoCA") following study entry; 20 centres agreed to contribute to this substudy. A MoCA score $<26$ was used to define cognitive impairment [26]. "Reverters" were defined as patients with an acute MoCA score $<26$, who demonstrated an improvement of $\geq 2$ points at 12 months, and patients with an acute MoCA score $<26$ who did not show this improvement were defined as "non-reverters". These thresholds are based on definitions from previously published work [27, 34].

\section{Imaging}

Imaging was undertaken locally at each study centre in accordance with a standardised protocol including axial T2, T2*-GRE, diffusion-weighted imaging, coronal T1 and FLAIR images [7]. Sequence parameters were specified for T2*-GRE [7]; the remaining sequences were obtained according to local protocols.

Neuroimaging analysis was carried out by two clinical research associates trained in rating structural markers of small-vessel disease and blinded to clinical details. Each structural marker was rated by a single rater, and all structural markers of cerebral small-vessel disease were rated in accordance with consensus criteria [40], and, where possible, the hemisphere contralateral to the acute stroke was preferentially counted.

Previous cortical infarcts were identified using T2 and FLAIR sequences and confirmed as non-acute through comparison with diffusion-weighted images (DWI). Lacunes were identified and counted on T2 and FLAIR sequences using definitions from the STRIVE criteria [40]. White-matter hyperintensities in deep (dWMH) and periventricular (pvWMH) distributions were rated on T2 and FLAIR sequences using the Fazekas scale [11, 12]. MRI-visible perivascular spaces in the basal ganglia (BG-PVS) and centrum semi-ovale (CSO-PVS) were rated on T2 and FLAIR sequences using a previously described validated visual rating scale [9, 22]. Medial temporal atrophy (MTA) was rated on T1 or FLAIR coronal images using the Scheltens visual scale [15]. Global cortical atrophy (GCA) was rated with the Pasquier scale [28] using axial T1, FLAIR or inverted T2 images. Cortical superficial siderosis (cSS) was identified on T2*-GRE sequences and classified as either focal, involving three or fewer sulci, or disseminated, involving four or more sulci [5]. Cerebral microbleeds (CMB) were rated using T2*-GRE sequences using the Microbleed Anatomical Rating Scale (MARS) [13]. Composite SVD [36,
37] and CAA [6] scores were determined using previously described scales.

Acute DWI lesions were defined as areas bright on the B1000 and dark on the corresponding ADC map; the side of the lesion, presence of single or multiple acute lesions, and evidence of cortical involvement were recorded. Evidence of haemorrhagic transformation was rated using the ECASS classification [32] using T2*-GRE sequences.

\section{Statistics}

We compared baseline clinical, demographic and imaging findings in patients with and without MoCA-defined cognitive impairment at 12 months, and for reverters compared with non-reverters. For all continuous variables, data were reviewed for normality, and if normally distributed, the independent $t$-test was used. If variables were ordinal or not normally distributed, the non-parametric Mann-Whitney $U$ test was used. Chi-squared or Fisher's exact tests were used for categorical variables. Performances of acute and 12 month MoCA were compared using paired $t$ tests (mean scores) or McNemar's test (proportion impaired).

The results of univariate comparisons were used to identify variables for inclusion in multivariable logistic regression models; all variables with $p<0.20$ were included in the adjusted analyses except for situations where variables both described the same phenomenon (for example, clinical history of previous ischaemic events and imaging evidence of a previous cortical infarct). Adjusted models considered only a single neuroimaging marker at a time. Given that these analyses were exploratory, we did not make an adjustment for multiple testing.

Statistical analysis was performed (GB) using Stata (Version 11.2).

\section{Results}

\section{Participants}

383 patients were recruited from the 20 sites participating in the MoCA substudy: 286 patients were eligible for this substudy, of whom 117 had 12 month cognitive data available (Fig. 1). The characteristics of those with 12 month MoCA data and those without (i.e. those excluded from this substudy) are shown in Table 1 . Those included within the analysis had a mean age of 73.1 years; $45(38.5 \%)$ were female, and the median NIHSS on admission was 3.5.

\section{Comparison of acute and 12 months MoCA performance}

Acute MoCA data were available for 114 patients with 12 month MoCA data (Fig. 2). The median time to acute 
Table 1 Baseline characteristics of included and excluded patients

\begin{tabular}{llll}
\hline & Included $(n=117)$ & Excluded $(n=169)$ & $p$ value \\
\hline Age (years), mean (SD) & $73.1(9.1)$ & $74.2(10.7)$ & 0.3757 \\
Sex, female, $n(\%)$ & $45(38.5)$ & $69(40.8)$ & 0.688 \\
Hypertension, $n(\%)$ & $60(52.2)$ & $100(59.2)$ & 0.243 \\
Hypercholesterolaemia, $n(\%)$ & $51(44.4)$ & $83(49.7)$ & 0.376 \\
Diabetes mellitus, $n(\%)$ & $11(9.5)$ & $33(19.5)$ & 0.021 \\
Smoking & & & \\
Never & $51(44.0)$ & $77(45.6)$ & 0.960 \\
Ex-smoker & $54(46.6)$ & $77(45.6)$ & \\
Current smoker & $11(9.5)$ & $15(8.9)$ & 0.569 \\
Heart failure, $n$ (\%) & $4(3.5)$ & $9(5.4)$ & 0.027 \\
AF prior to study entry, $n(\%)$ & $28(23.9)$ & $61(36.3)$ & 0.0288 \\
Educational age (years), mean (SD) & $16.8(3.5)$ & $16.0(2.5)$ & 0.0210 \\
NIHSS, median (IQR) & $3.5(2-9)$ & $5.5(2-11)$ & 0.0091 \\
Acute MoCA score, median (IQR) & $25(21-27)$ & $23(18-26)$ & 0.0321 \\
Discharge mRS, median (IQR) & $1(0-2)$ & $1(1-3)$ & 0.941 \\
Further intracerebral event within & $6(5.1)$ & $9(5.3)$ & \\
$\quad 12$ months of study entry, $n(\%)$ & & & \\
\hline
\end{tabular}

Percentage values were calculated using the total number of patients for whom data were available as the denominator. $p$ values are from independent t-tests (age, educational age, discharge mRS), Mann-Whitney $U$ test (NIHSS, acute MoCA score), Fisher's exact test (heart failure) or Chi-squared tests (remainder)

$A F$ Atrial fibrillation, IQR interquartile range, MoCA Montreal cognitive assessment, $m R S$ modified Rankin scale, NIHSS National Institutes of Health Stroke Scale, SD standard deviation

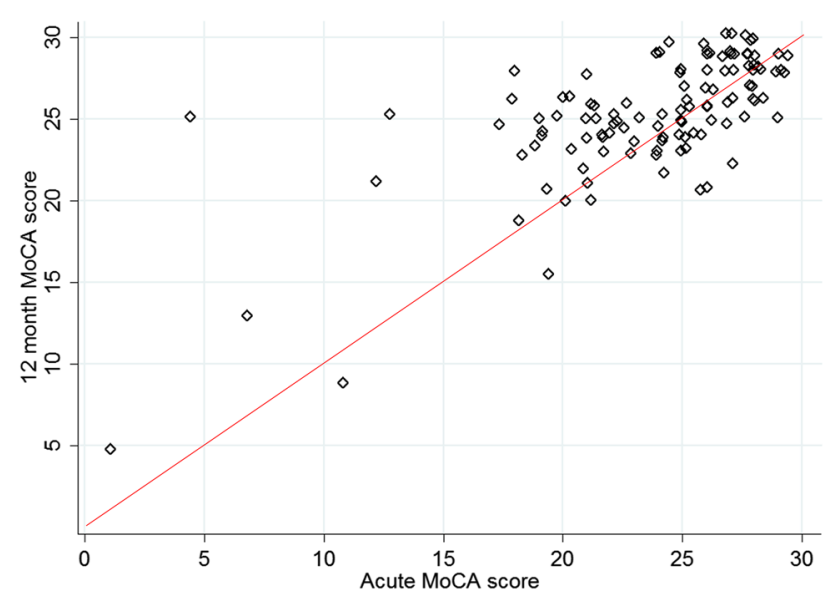

Fig. 2 Distribution of acute and 12 months MoCA scores. Each patient is shown by a single diamond; the data have been jittered to show individual points. The line of equality is shown in red

MoCA assessment was 4 days (IQR 2-8 days). Overall, there was an improvement at 12 months (mean difference 1.69 points where maximum score is $30, p<0.00001$; Table 2). Scores improved across all subdomains except for attention (which showed a deterioration) and were statistically significant for visuo-executive function (mean difference 0.23 points, $p=0.0470$ ), abstraction (mean difference 0.14 points, $p=0.0176$ ) and delayed recall (mean difference 0.62 points, $p=0.0002)$.
We also considered whether the proportion of participants impaired across domains (defined as scoring less than full marks) changed with time (Table 2). Fewer patients demonstrated MoCA impairments at 12 months $(51.3 \%$ vs $57.9 \%$, $p=0.0719$ ), and there were lower proportions of impaired participants across all domains; this was statistically significant for language $(48.7 \%$ vs $59.7 \%, p=0.0269)$, and abstraction $(22.2 \%$ vs $31.6 \%, p=0.0233)$.

\section{Clinical and imaging associations of cognitive impairment 12 months following index ischaemic event}

In our cohort, $51.3 \%(n=60)$ had an abnormal MoCA score $(<26)$ at 12 months. When comparing those with and without MoCA-defined cognitive impairment at 12 months, those with impairment were older (mean age 75.6 years vs 70.5 years, $p=0.0022$ ), had fewer years of education (mean 15.9 years vs 17.7 years, $p=0.0077$ ), had a higher admission NIHSS (median score 5.5 vs $2, p=0.0060$ ), lower acute MoCA score (median 22 vs 27, $p<0.00001$ ) and higher discharge $\mathrm{mRS}$ (median score 2 vs $1, p=0.0004$ ). In a multivariable logistic regression analysis including these variables, only acute MoCA score remained associated with MoCA impairment at 12 months (per-point increase, OR 0.73, 95\% CI 0.59-0.91, $p=0.005$ ).

Patients with cognitive impairment at 12 months had higher grades of pvWMH (IQR $0-1$ vs $0-0, p=0.0545$ ) and 
Table 2 Comparison of MoCA performance acutely (median 4 days following ischaemic event) and at 12 months

\begin{tabular}{|c|c|c|c|c|c|c|c|c|}
\hline & \multicolumn{5}{|c|}{ Comparison of means } & \multicolumn{3}{|c|}{ Comparison of proportions } \\
\hline & $\begin{array}{l}\text { Maximum } \\
\text { achievable } \\
\text { score }\end{array}$ & $\begin{array}{l}\text { Acute MoCA } \\
(n=114) ; \text { mean } \\
\text { score (SD) }\end{array}$ & $\begin{array}{l}12 \text { month } \\
\text { MoCA } \\
(n=117) ; \text { mean } \\
\text { score (SD) }\end{array}$ & $\begin{array}{l}\text { Mean difference } \\
(95 \% \mathrm{CI})\end{array}$ & $p$ value & $\begin{array}{l}\text { Acute MoCA } \\
(n=114) ; \\
\text { impaired, } n(\%)\end{array}$ & $\begin{array}{l}12 \text { month } \\
\text { MoCA } \\
(n=117), \\
\text { impaired, } n(\%)\end{array}$ & $p$ value \\
\hline Total score & 30 & $23.55(4.95)$ & $25.25(3.88)$ & $1.69(1.03-2.36)$ & $<0.00001$ & $66(57.9)$ & $60(51.3)$ & 0.0719 \\
\hline Visuo-executive & 5 & $3.77(1.38)$ & $4.00(1.13)$ & $0.23(0.00-0.45)$ & 0.0470 & $72(63.2)$ & $69(59.0)$ & 0.2888 \\
\hline Naming & 3 & $2.75(0.61)$ & $2.82(0.49)$ & $\begin{array}{l}0.07(-0.05 \text { to } \\
0.19)\end{array}$ & 0.2399 & $22(19.3)$ & $17(14.5)$ & 0.3173 \\
\hline Attention & 6 & $4.98(1.49)$ & $4.70(1.60)$ & $\begin{array}{l}-0.28(-0.05 \text { to } \\
0.61)\end{array}$ & 0.0964 & $61(53.5)$ & $54(46.2)$ & 0.1228 \\
\hline Orientation & 6 & $5.58(1.06)$ & $5.71(0.73)$ & $\begin{array}{l}0.13(-0.05 \text { to } \\
0.32)\end{array}$ & 0.1628 & $25(21.9)$ & $24(20.5)$ & 0.5050 \\
\hline Language & 3 & $2.11(0.91)$ & $2.25(0.96)$ & $\begin{array}{l}0.13(-0.05 \text { to } \\
0.32)\end{array}$ & 0.1591 & $68(59.7)$ & $57(48.7)$ & 0.0269 \\
\hline Abstraction & 2 & $1.58(0.68)$ & $1.72(0.59)$ & $0.14(0.02-0.26)$ & 0.0176 & $36(31.6)$ & $26(22.2)$ & 0.0233 \\
\hline Delayed recall & 5 & $2.34(1.62)$ & $2.96(1.53)$ & $0.62(0.31-0.94)$ & 0.0002 & $102(89.5)$ & $98(83.8)$ & 0.1444 \\
\hline
\end{tabular}

Impairment was defined as scoring less than full marks in a given domain; MoCA impairment was defined as previously (score $<26)$. Percentage values were calculated using the total number of patients for whom data were available as the denominator. For the comparison of mean scores, $p$ values are from paired $t$ tests, and for the comparison of proportion impaired, McNemar's test was used

CI Confidence intervals, MoCA Montreal Cognitive Assessment, $S D$ standard deviation

had higher CAA scores (median score 0.5 vs $0, p=0.0005$ ). There were no statistically significant differences between the two groups in the imaging features of the index ischaemic lesion (presence of acute DWI lesion at study entry, side of index lesion, presence of multiple index lesions on DWI, presence of a cortical lesion, evidence of haemorrhagic transformation). In adjusted analyses (adjusted for age, educational age, discharge mRS and acute MoCA score), cognitive impairment at 12 months remained associated with CAA score (per-point increase, OR 4.09, 95\% CI 1.36-12.33, $p=0.012$ ) but not pvWMH grade (OR 1.15, 95\% CI 0.54-2.44, $p=0.725$ ).

\section{MoCA trajectory}

In this cohort, 66 patients had an acute MoCA score below 26; of these, 59.1\% $(n=39)$ were "reverters" (Fig. 3). Nonreverters had higher acute MoCA scores (median 24 vs 21, $p=0.0002$ ) and lower 12-month MoCA scores (median 23 vs $25, p=0.0008$ ); there were no other clinical or demographic (including years of education) differences between the two groups.

The imaging characteristics of reverters and non-reverters are shown in Table 3. Non-reverters had lower baseline pvWMH grade (IQR 0-0 vs $0-1, p=0.0752$ ), but higher CSO-PVS grade (median grade 2 vs $1, p=0.0306$ ), and were more likely to have cerebral microbleeds $(22.2 \%$ vs $2.6 \%, p=0.016)$ and in particular, strictly lobar microbleeds $(14.8 \%$ vs $0.0 \%, p=0.024)$. Non-reverters also had a higher

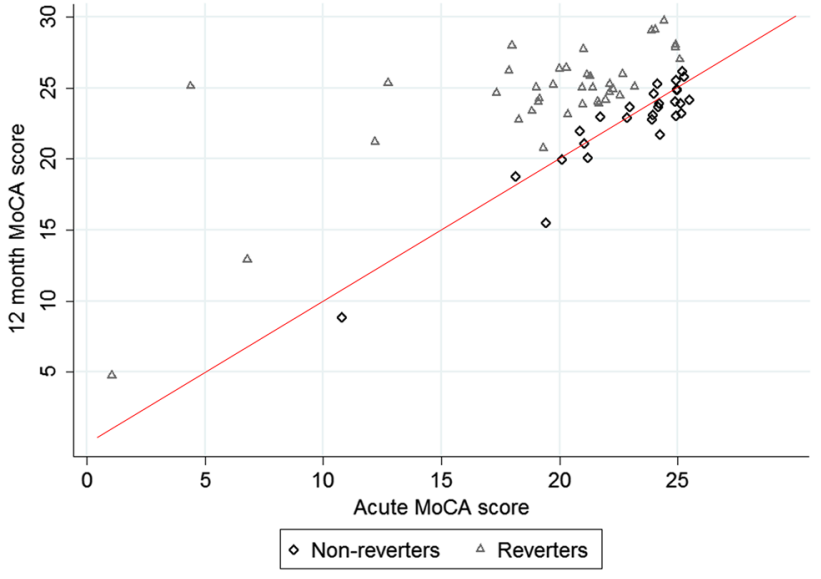

Fig. 3 Distribution of acute and 12 months MoCA scores for reverters and non-reverters. Each patient is shown by a single symbol, as indicated by the key; the data have been jittered to show individual points. The line of equality is shown in red

composite SVD (mean 0.88 vs $0.27, p=0.0046$ ) and CAA (mean 0.80 vs $0.25, p=0.0007$ ) scores. In unadjusted logistic regression analyses (Table 4$)$, non-reversion remained positively associated with CSO-PVS grade (per-grade increase, OR 1.83, $p=0.029)$, cerebral microbleed presence (OR 10.86, $p=0.032$ ), SVD score (per-point increase, OR 2.91, $p=0.015$ ) and CAA score (per-point increase, OR $6.71, p=0.001)$, and negatively associated with the presence of multiple lesions at study entry (OR 0.11, $p=0.040$ ). 
Table 3 Comparison of imaging characteristics of reverters vs non-reverters
Table 4 Unadjusted and adjusted logistic regression analyses for predictors of nonreversion

\begin{tabular}{|c|c|c|c|}
\hline & Reverters & Non-reverters & $p$ value \\
\hline$n(\%)$ & $39(59.1)$ & $27(40.9)$ & - \\
\hline \multicolumn{4}{|l|}{ Structural imaging markers at study entry } \\
\hline Imaging evidence of previous cortical infarct, $n(\%)$ & $3(7.7)$ & $4(14.8)$ & 0.432 \\
\hline Lacunes, presence, $n(\%)$ & $5(12.8)$ & $5(20.8)$ & 0.485 \\
\hline pvWMH grade, median (IQR) & $0(0-1)$ & $0(0-0)$ & 0.0752 \\
\hline dWMH grade, median (IQR) & $1(0-1)$ & $0(0-1)$ & 0.3217 \\
\hline CSO-PVS grade, median (IQR) & $1(1-2)$ & $2(1-3)$ & 0.0306 \\
\hline BG-PVS grade, median (IQR) & $1(1-1)$ & $1(1-1)$ & 0.1221 \\
\hline MTA grade, median (IQR) & $1(0-1)$ & $1(0-1)$ & 0.7561 \\
\hline GCA grade, median (IQR) & $1(0-1)$ & $1(0-1)$ & 0.9943 \\
\hline CMB, presence, $n(\%)$ & $1(2.6)$ & $6(22.2)$ & 0.016 \\
\hline Strictly lobar CMB, $n(\%)$ & $0(0.0)$ & $4(14.8)$ & 0.024 \\
\hline \multicolumn{4}{|l|}{ Composite SVD score, median (IQR) } \\
\hline Median (IQR) & $0(0-0)$ & $1(0-1)$ & \multirow[t]{2}{*}{0.0046} \\
\hline Mean (SD) & $0.27(0.61)$ & $0.88(0.93)$ & \\
\hline \multicolumn{4}{|l|}{ Composite CAA score, median (IQR) } \\
\hline Median (IQR) & $0(0-0.5)$ & $1(0-1)$ & \multirow[t]{2}{*}{0.0007} \\
\hline Mean (SD) & $0.25(0.44)$ & $0.80(0.62)$ & \\
\hline \multicolumn{4}{|l|}{ Imaging features of index ischaemic event } \\
\hline Acute DWI lesion at study entry, $n(\%)$ & $32(82.1)$ & $19(76.0)$ & 0.557 \\
\hline \multicolumn{4}{|l|}{ Side of index lesion, $n(\%)$} \\
\hline Left & $11(35.5)$ & $6(31.6)$ & \multirow[t]{3}{*}{0.249} \\
\hline Right & $16(51.6)$ & $13(68.4)$ & \\
\hline Bilateral & $4(12.9)$ & $0(0.0)$ & \\
\hline Presence of multiple lesions, $n(\%)$ & $11(34.4)$ & $1(5.3)$ & 0.020 \\
\hline Cortical lesion, $n(\%)$ & $21(65.6)$ & $14(73.7)$ & 0.756 \\
\hline Evidence of haemorrhagic transformation, $n(\%)$ & $8(20.5)$ & $2(8.7)$ & 0.298 \\
\hline
\end{tabular}

Percentage values were calculated using the total number of patients for whom data werer available as the denominator. $p$ values are from Mann-Whitney $U$ tests (where median and IQR are given), Chi-squared tests (acute DWI lesion at study entry) or Fisher's exact test (remainder)

$B G$ - $P V S$ MRI-visible perivascular spaces in the basal ganglia, $C M B$ cerebral microbleed, $C S O$ MRI-visible perivascular spaces in the centrum semi-ovale, $D W I$ diffusion-weighted imaging, $d W M H$ deep white-matter hyperintensities, GCA global cortical atrophy, IQR interquartile range, $M o C A$ Montreal cognitive assessment, $M T A$ medial temporal atrophy, $p v W V H$ periventricular hyperintensities, $S D$ standard deviation

\begin{tabular}{lllllll}
\hline & Unadjusted & & & \multicolumn{2}{l}{$\begin{array}{l}\text { Adjusted for acute MoCA } \\
\text { score }\end{array}$} \\
\cline { 2 - 3 } \cline { 6 - 7 } & & & & & \\
& OR $(95 \%$ CI $)$ & & $p$ value & & OR (95\% CI) & $p$ value \\
\hline pvWMH (per-grade increase) & $0.41(0.12-1.35)$ & 0.143 & & $0.50(0.15-1.68)$ & 0.264 \\
CSO-PVS (per-grade increase) & $1.83(1.06-3.15)$ & 0.029 & & $1.96(1.05-3.66)$ & 0.035 \\
BG-PVS (per-grade increase) & $2.60(0.74-9.19)$ & 0.137 & & $2.26(0.63-8.02)$ & 0.209 \\
CMB (presence) & $10.86(1.22-96.34)$ & 0.032 & & $9.36(0.92-95.34)$ & 0.059 \\
SVD score (per-point increase) & $2.91(1.23-6.88)$ & 0.015 & & $2.47(1.02-6.00)$ & 0.046 \\
CAA score (per-point increase) & $6.71(2.10-21.50)$ & 0.001 & & $6.70(1.88-23.98)$ & 0.003 \\
Presence of multiple lesions at study entry & $0.11(0.02-0.90)$ & 0.040 & & $0.11(0.01-1.01)$ & 0.051 \\
\hline
\end{tabular}

Each model considered one imaging marker at a time

$B G$-PVS MRI-visible perivascular spaces in the centrum semi-ovale, $C A A$ cerebral amyloid angiopathy, $C I$ confidence interval, $C M B$ cerebral microbleed, CSO-PVS MRI-visible perivascular spaces in the centrum semi-ovale, MoCA Montreal cognitive assessment, $p v W M H$ periventricular hyperintensities, SVD smallvessel disease 
Similar associations were observed in analyses adjusted for MoCA score (Table 4).

\section{Discussion}

MoCA-defined cognitive impairment at 12 months is common, observed in approximately half of our cohort, and associated with factors relating to brain resilience (age, educational age) and stroke severity (acute MoCA score, NIHSS, discharge mRS), as well as increases in a composite CAA score. Overall, we found that MoCA performance at 12 months improves compared with acute performance, and we show that the presence of structural imaging markers of small-vessel disease (CSO-PVS, cerebral microbleeds, composite SVD and CAA scores) is associated with non-reversion.

Our use of composite scores for the two most common small-vessel diseases provides new perspectives on the small-vessel mechanisms which might underlie poststroke dementia. Composite scores are hypothesised to better reflect overall pathological burden and have shown associations with a number of clinical measures including cognitive performance $[8,18,35,36,39]$. In our study, we observed an association of 12-month cognitive performance with the composite CAA score. This score includes nonhaemorrhagic markers of CAA such as CSO-PVS and WMH which do not feature in the current diagnostic criteria for CAA [21], and which might have more relevance in nonhaemorrhagic patient populations. The association of CAA with dementia following intracerebral haemorrhage has been described [25], as have associations between strictly lobar microbleeds and executive function in patients with ischaemic stroke or TIA [14]. Whilst our finding that CAA score is associated with 12-month MoCA impairment should be interpreted with caution, given the low prevalence of haemorrhagic markers and the relatively small size of the cohort, it might provide further evidence that imaging markers of CAA are associated with cognitive performance beyond intracerebral haemorrhage, a finding already observed in non-haemorrhagic memory clinic populations [33, 42].

Our observation that overall cognitive performance can improve with time following an ischaemic event is in keeping with data from previous studies $[2,10,17,19$, 29], as are the significant domain specific improvements in visuo-executive function, abstraction and delayed recall $[27,34]$. As well as improvements in raw scores, we also found that levels of impairment were lower at 12 months for most domains. However, we did note that for a number of domains, whilst raw scores improved significantly, patients remained impaired range (as we defined it). This highlights the difficulties in quantifying deficits when considering individual domains.
We also describe the characteristics of patients with impaired acute performance who demonstrate an improvement of two or more points - so-called "reverters" [27, 34] and those who do not. Whilst use of the term "reverter" has been criticised for suggesting that cognitive performance returns to normal [27], it is useful as a standardised method for defining improvement. Whilst we did not find any clinical or demographic differences between reverters and nonreverters (except for those relating to MoCA scores), there were imaging differences. Non-reverters appeared to have more evidence of small-vessel disease (CSO-PVS, cerebral microbleeds, and higher composite SVD and CAA scores), and were less likely to have had multiple acute DWI lesions at study entry. As discussed above, the association between both multiple lesions and lower acute MoCA scores amongst the reverters might suggest that multiple lesions are more likely to result in an acute reversible cognitive impairment-although the acute disturbance in this cohort does not seem to be typical for delirium, given the lack of attentional improvement with time. Replication of this work in larger cohorts will be important for confirming and better quantifying these observations.

The strengths of this study are its multicentre prospective design, and the detailed clinical and radiological descriptions available for the study participants. However, there are also some limitations. Firstly, only a subset of centres collected 12-month MoCA data, and even within these centres MoCA data were not collected for all potentially eligible participants. The excluded patients had more comorbidities, lower educational age, more severe ischaemic events (as defined by NIHSS and discharge mRS) and had lower acute MoCA scores, all of which are associated with poorer cognitive outcome at 12 months. Given this, and the fact that our cohort tended to have milder strokes (median NIHSS 3.5), the cognitive performance of our cohort might be better than expected and not representative of all cardioembolic ischaemic stroke or TIA cohorts. Additionally, we were unable to account for patients who were aphasic; this should be considered in the interpretation of our results. We note that group sizes for some analyses are small and the prevalence of haemorrhagic markers in our cohort was low; in view of this, these results should be interpreted cautiously. Our modest group sizes also precluded further subdivision into the reverter and non-reverter groups; in particular, a comparison of non-reverters with stable cognition vs those demonstrating further decline would be of interest, and an important area for future work. Cognitive assessments acutely after an ischaemic event period can be difficult to interpret, as cognitive performance can be influenced by intercurrent illness (such as coexisting infection, as one example) or complications relating to the ischaemic event itself (for example, seizures). Whilst we acknowledge that a slightly 
delayed assessment (for example, 2-4 weeks after an ischaemic event), might avoid these complicating factors, in routine clinical practice the first measurement of cognitive performance (using the MoCA and other tools) is often in the acute period, as in our study; the implications of early assessments are therefore of relevance. Finally, we only used a single measure (the MoCA) to estimate cognitive performance at a single time point; the MoCA has some intrinsic limitations, including the fact that it is primarily a screening tool and thus likely underestimates the severity and breadth of cognitive impairment that result from stroke [3, 4]. The absence of data on MoCA performance at additional time points, both before 1 year (for example, at 3 or 6 months) and beyond this (for example, at 2 years), is a further limitation; this would allow us to better define post-event cognitive trajectory, and the lack of assessment at additional time points is a limitation of our study design. Nevertheless, the positive findings and associations found show some promise that it might be a useful tool for monitoring for change over time.

We conclude that MoCA-defined cognitive impairment 12 months following an ischaemic event is common, and that structural imaging features of small-vessel disease appear associated both with deficits and a lack of improvement at 12 months. Further work that clarifies the role of small-vessel diseases in this context will be essential for refining future cognitive rehabilitation strategies.

Acknowledgements The CROMIS-2 collaborators: Rustam Al-Shahi Salman, Louise Shaw, Kirsty Harkness, Jane Sword, Azlisham Mohd Nor, Pankaj Sharma, Deborah Kelly, Frances Harrington, Marc Randall, Matthew Smith, Karim Mahawish, Abduelbaset Elmarim, Bernard Esisi, Claire Cullen, Arumug Nallasivam, Christopher Price, Adrian Barry, Christine Roffe, John Coyle, Ahamad Hassan, Caroline Lovelock, Jonathan Birns, David Cohen, L. Sekaran, Adrian Parry-Jones, Anthea Parry, David Hargroves, Harald Proschel, Prabel Datta, Khaled Darawil, Aravindakshan Manoj, Mathew Burn, Chris Patterson, Elio Giallombardo, Nigel Smyth, Syed Mansoor, Ijaz Anwar, Rachel Marsh, Sissi Ispoglou, Dinesh Chadha, Mathuri Prabhakaran, Sanjeevikumar Meenakishundaram, Janice O'Connell, Jon Scott, Vinodh Krishnamurthy, Prasanna Aghoram, Michael McCormick, Paul O'Mahony, Martin Cooper, Lillian Choy, Peter Wilkinson, Simon Leach, Sarah Caine, Ilse Burger, Gunaratam Gunathilagan, Paul Guyler, Hedley Emsley, Michelle Davis, Dulka Manawadu, Kath Pasco, Maam Mamun, Robert Luder, Mahmud Sajid, Ijaz Anwar, James Okwera, Julie Staals, Elizabeth Warburton, Kari Saastamoinen, Timothy England, Janet Putterill, Enrico Flossman, Michael Power, Krishna Dani, David Mangion, Appu Suman, John Corrigan, Enas Lawrence, Djamil Vahidassr.

Funding The CROMIS-2 study is funded by the Stroke Association and British Heart Foundation. GB holds an NIHR Academic Clinical Fellowship, and received funding from the Rosetrees Trust. GA receives funding from the NIHR University College London Hospitals Biomedical Research Centre. RASS is funded by an MRC senior clinical fellowship. MMB's Chair in Stroke Medicine is supported by the Reta Lila Weston Trust for Medical Research. DJW receives research support from the Stroke Association, the British Heart Foundation and the Rosetrees Trust. This work was undertaken at UCLH/UCL which receives a proportion of funding from the Department of Health's NIHR Biomedical Research Centres funding scheme.

\section{Compliance with ethical standards}

Conflicts of interest $\mathrm{HC}$ has received institutional research support from Bayer; honoraria for lectures and an Advisory Board from Bayer, diverted to a local charity; and travel/accommodation expenses for participation in scientific meetings covered by Bayer. GYHL acts as a consultant for Bayer/Janssen, BMS/Pfizer, Medtronic, Boehringer Ingelheim, Novartis, Verseon and Daiichi-Sankyo, and as a speaker for Bayer, BMS/Pfizer, Medtronic, Boehringer Ingelheim, and DaiichiSankyo; no fees are directly received personally. The remaining authors report no disclosures or conflicts of interest relevant to the manuscript.

Open Access This article is distributed under the terms of the Creative Commons Attribution 4.0 International License (http://creativeco mmons.org/licenses/by/4.0/), which permits unrestricted use, distribution, and reproduction in any medium, provided you give appropriate credit to the original author(s) and the source, provide a link to the Creative Commons license, and indicate if changes were made.

\section{References}

1. Altieri M, Di Piero V, Pasquini M, Gasparini M, Vanacore N, Vicenzini E, Lenzi GL (2004) Delayed poststroke dementia: a 4-year follow-up study. Neurology 62:2193-2197

2. Ballard C, Rowan E, Stephens S, Kalaria R, Kenny RA (2003) Prospective follow-up study between 3 and 15 months after stroke: improvements and decline in cognitive function among dementiafree stroke survivors $>75$ years of age. Stroke 34:2440-2444

3. Chan E, Altendorff S, Healy C, Werring DJ, Cipolotti L (2017) The test accuracy of the Montreal Cognitive Assessment (MoCA) by stroke lateralisation. J Neurol Sci 373:100-104

4. Chan E, Khan S, Oliver R, Gill SK, Werring DJ, Cipolotti L (2014) Underestimation of cognitive impairments by the Montreal Cognitive Assessment (MoCA) in an acute stroke unit population. J Neurol Sci 343:176-179

5. Charidimou A, Linn J, Vernooij MW, Opherk C, Akoudad S, Baron JC, Greenberg SM, Jager HR, Werring DJ (2015) Cortical superficial siderosis: detection and clinical significance in cerebral amyloid angiopathy and related conditions. Brain 138:2126-2139

6. Charidimou A, Martinez-Ramirez S, Reijmer YD, Oliveira-Filho J, Lauer A, Roongpiboonsopit D, Frosch M, Vashkevich A, Ayres A, Rosand J, Gurol ME, Greenberg SM, Viswanathan A (2016) Total magnetic resonance imaging burden of small vessel disease in cerebral amyloid angiopathy: an imaging-pathologic study of concept validation. JAMA Neurol 73:994-1001

7. Charidimou A, Wilson D, Shakeshaft C, Ambler G, White M, Cohen H, Yousry T, Al-Shahi Salman R, Lip G, Houlden H, Jager HR, Brown MM, Werring DJ (2015) The Clinical Relevance of Microbleeds in Stroke study (CROMIS-2): rationale, design, and methods. Int J Stroke 100:155-161

8. Del Brutto VJ, Ortiz JG, Del Brutto OH, Mera RM, Zambrano M, Biller J (2018) Total cerebral small vessel disease score and cognitive performance in community-dwelling older adults. Results from the Atahualpa Project. Int J Geriatr Psychiatry 33:325-331

9. Doubal FN, MacLullich AM, Ferguson KJ, Dennis MS, Wardlaw JM (2010) Enlarged perivascular spaces on MRI are a feature of cerebral small vessel disease. Stroke 41:450-454 
10. Douiri A, Rudd AG, Wolfe CD (2013) Prevalence of poststroke cognitive impairment: South London Stroke Register 1995-2010. Stroke 44:138-145

11. Fazekas F, Chawluk JB, Alavi A, Hurtig HI, Zimmerman RA (1987) MR signal abnormalities at $1.5 \mathrm{~T}$ in Alzheimer's dementia and normal aging. AJR Am J Roentgenol 149:351-356

12. Fazekas F, Kleinert R, Offenbacher H, Schmidt R, Kleinert G, Payer F, Radner H, Lechner H (1993) Pathologic correlates of incidental MRI white matter signal hyperintensities. Neurology 43:1683-1689

13. Gregoire SM, Chaudhary UJ, Brown MM, Yousry TA, Kallis C, Jager HR, Werring DJ (2009) The Microbleed Anatomical Rating Scale (MARS): reliability of a tool to map brain microbleeds. Neurology 73:1759-1766

14. Gregoire SM, Scheffler G, Jager HR, Yousry TA, Brown MM, Kallis C, Cipolotti L, Werring DJ (2013) Strictly lobar microbleeds are associated with executive impairment in patients with ischemic stroke or transient ischemic attack. Stroke 44:1267-1272

15. Harper L, Barkhof F, Fox NC, Schott JM (2015) Using visual rating to diagnose dementia: a critical evaluation of MRI atrophy scales. J Neurol Neurosurg Psychiatry 86:1225-1233

16. Harrison JK, Fearon P, Noel-Storr AH, McShane R, Stott DJ, Quinn TJ (2015) Informant Questionnaire on Cognitive Decline in the Elderly (IQCODE) for the diagnosis of dementia within a secondary care setting. Cochrane Datab Syst Rev 3:CD010772

17. Hochstenbach JB, den Otter R, Mulder TW (2003) Cognitive recovery after stroke: a 2-year follow-up. Arch Phys Med Rehabil 84:1499-1504

18. Huijts M, Duits A, van Oostenbrugge RJ, Kroon AA, de Leeuw PW, Staals J (2013) Accumulation of MRI markers of cerebral small vessel disease is associated with decreased cognitive function. A study in first-ever lacunar stroke and hypertensive patients. Front Aging Neurosci 5:72

19. Hurford R, Charidimou A, Fox Z, Cipolotti L, Werring DJ (2013) Domain-specific trends in cognitive impairment after acute ischaemic stroke. J Neurol 260:237-241

20. Ihara M, Washida K (2018) Linking atrial fibrillation with Alzheimer's disease: epidemiological, pathological, and mechanistic evidence. J Alzheimers Dis 62:61-72

21. Linn J, Halpin A, Demaerel P, Ruhland J, Giese AD, Dichgans M, van Buchem MA, Bruckmann H, Greenberg SM (2010) Prevalence of superficial siderosis in patients with cerebral amyloid angiopathy. Neurology 74:1346-1350

22. Maclullich AM, Wardlaw JM, Ferguson KJ, Starr JM, Seckl JR, Deary IJ (2004) Enlarged perivascular spaces are associated with cognitive function in healthy elderly men. J Neurol Neurosurg Psychiatry 75:1519-1523

23. Mok VC, Lam BY, Wong A, Ko H, Markus HS, Wong LK (2017) Early-onset and delayed-onset poststroke dementia—revisiting the mechanisms. Nat Rev Neurol 13:148-159

24. Mok VCT, Lam BYK, Wang Z, Liu W, Au L, Leung EYL, Chen S, Yang J, Chu WCW, Lau AYL, Chan AYY, Shi L, Fan F, Ma SH, Ip V, Soo YOY, Leung TWH, Kwok TCY, Ho CL, Wong LKS, Wong A (2016) Delayed-onset dementia after stroke or transient ischemic attack. Alzheimer's Dementia 12:1167-1176

25. Moulin S, Labreuche J, Bombois S, Rossi C, Boulouis G, Henon H, Duhamel A, Leys D, Cordonnier C (2016) Dementia risk after spontaneous intracerebral haemorrhage: a prospective cohort study. Lancet Neurol 15:820-829

26. Nasreddine ZS, Phillips NA, Bedirian V, Charbonneau S, Whitehead V, Collin I, Cummings JL, Chertkow H (2005) The Montreal Cognitive Assessment, MoCA: a brief screening tool for mild cognitive impairment. J Am Geriatr Soc 53:695-699

27. Nijsse B, Visser-Meily JM, van Mierlo ML, Post MW, de Kort PL, van Heugten CM (2017) Temporal evolution of poststroke cognitive impairment using the montreal cognitive assessment. Stroke 48:98-104

28. Pasquier F, Leys D, Weerts JG, Mounier-Vehier F, Barkhof F, Scheltens P (1996) Inter- and intraobserver reproducibility of cerebral atrophy assessment on MRI scans with hemispheric infarcts. Eur Neurol 36:268-272

29. Patel M, Coshall C, Rudd AG, Wolfe CD (2003) Natural history of cognitive impairment after stroke and factors associated with its recovery. Clin Rehabil 17:158-166

30. Pendlebury ST, Rothwell PM (2009) Prevalence, incidence, and factors associated with pre-stroke and post-stroke dementia: a systematic review and meta-analysis. Lancet Neurol 8:1006-1018

31. Poggesi A, Inzitari D, Pantoni L (2015) Atrial fibrillation and cognition: epidemiological data and possible mechanisms. Stroke 46:3316-3321

32. Renou P, Sibon I, Tourdias T, Rouanet F, Rosso C, Galanaud D, Drier A, Coudert M, Deltour S, Crozier S, Dormont D, Samson Y (2010) Reliability of the ECASS radiological classification of postthrombolysis brain haemorrhage: a comparison of CT and three MRI sequences. Cerebrovasc Dis 29:597-604

33. Shams S, Martola J, Charidimou A, Cavallin L, Granberg T, Shams M, Forslin Y, Aspelin P, Kristoffersen-Wiberg M, Wahlund LO (2016) Cortical superficial siderosis: prevalence and biomarker profile in a memory clinic population. Neurology 87:1110-1117

34. Sivakumar L, Kate M, Jeerakathil T, Camicioli R, Buck B, Butcher K (2014) Serial montreal cognitive assessments demonstrate reversible cognitive impairment in patients with acute transient ischemic attack and minor stroke. Stroke 45:1709-1715

35. Song TJ, Kim J, Song D, Yoo J, Lee HS, Kim YJ, Nam HS, Heo JH, Kim YD (2017) Total cerebral small-vessel disease score is associated with mortality during follow-up after acute ischemic stroke. J Clin Neurol 13:187-195

36. Staals J, Booth T, Morris Z, Bastin ME, Gow AJ, Corley J, Redmond P, Starr JM, Deary IJ, Wardlaw JM (2015) Total MRI load of cerebral small vessel disease and cognitive ability in older people. Neurobiol Aging 36:2806-2811

37. Staals J, Makin SD, Doubal FN, Dennis MS, Wardlaw JM (2014) Stroke subtype, vascular risk factors, and total MRI brain smallvessel disease burden. Neurology 83:1228-1234

38. Tang WK, Chen YK, Lu JY, Wong A, Mok V, Chu WC, Ungvari GS, Wong KS (2011) Absence of cerebral microbleeds predicts reversion of vascular 'cognitive impairment no dementia' in stroke. Int J Stroke 6:498-505

39. Uiterwijk R, van Oostenbrugge RJ, Huijts M, De Leeuw PW, Kroon AA, Staals J (2016) Total cerebral small vessel disease MRI score is associated with cognitive decline in executive function in patients with hypertension. Front Aging Neurosci 8:301

40. Wardlaw JM, Smith EE, Biessels GJ, Cordonnier C, Fazekas F, Frayne R, Lindley RI, O’Brien JT, Barkhof F, Benavente OR, Black SE, Brayne C, Breteler M, Chabriat H, Decarli C, de Leeuw FE, Doubal F, Duering M, Fox NC, Greenberg S, Hachinski V, Kilimann I, Mok V, Oostenbrugge R, Pantoni L, Speck O, Stephan BC, Teipel S, Viswanathan A, Werring D, Chen C, Smith C, van Buchem M, Norrving B, Gorelick PB, Dichgans M, nEuroimaging STfRVco (2013) Neuroimaging standards for research into small vessel disease and its contribution to ageing and neurodegeneration. Lancet Neurol 12:822-838

41. Wilson D, Ambler G, Shakeshaft C, Brown MM, Charidimou A, Al-Shahi Salman R, Lip GYH, Cohen H, Banerjee G, Houlden H, White MJ, Yousry TA, Harkness K, Flossmann E, Smyth N, Shaw LJ, Warburton E, Muir KW, Jager HR, Werring DJ, collaborators C (2018) Cerebral microbleeds and intracranial haemorrhage risk in patients anticoagulated for atrial fibrillation after acute ischaemic stroke or transient ischaemic attack (CROMIS-2): a multicentre observational cohort study. Lancet Neurol 17:539-547 
42. Xiong L, Boulouis G, Charidimou A, Roongpiboonsopit D, Jessel MJ, Pasi M, Reijmer YD, Fotiadis P, Ayres A, Merrill E, Schwab K, Blacker D, Gurol ME, Greenberg SM, Viswanathan A (2018) Dementia incidence and predictors in cerebral amyloid angiopathy patients without intracerebral hemorrhage. J Cerebral Blood Flow Metab 38:241-249
43. Yoon JA, Kim DY, Sohn MK, Lee J, Lee SG, Lee YS, Han EY, Joo MC, Oh GJ, Han J, Park M, Park KP, Noh KH, Chang WH, Shin YI, Kim YH (2017) Factors associated with improvement or decline in cognitive function after an ischemic stroke in Korea: the Korean stroke cohort for functioning and rehabilitation (KOSCO) study. BMC Neurol 17:9 